\title{
Matching Information and Ambient Media
}

\author{
Albrecht Schmidt, Hans-W. Gellersen and Michael Beigl \\ Telecooperation Office, University of Karlsruhe \\ Vincenz-Prießnitz-Str. 1, 76131 Karlsruhe, Germany \\ \{albrecht, hwg, michael\}@teco.edu \\ http: //www.teco.edu/
}

\begin{abstract}
Ambient media have recently been introduced as a means to present information in our surrounding space in subtle and unmonopolizing ways, promoting peripheral awareness. This paper explores the issue of matching virtual information to ambient media, investigating ambient counterparts as natural matches and ambient links as user controlled matches. Ambient counterparts are media in our surroundings that have an intuitive and strong relationship with the virtual information they present, and we report on an application of such counterparts for comparative web site awareness employed for group motivation in their workplace. Ambient links are relation between ambient media and events in the virtual world actively assigned by the user for ambient notification. We describe two tools for creating ambient links, the first one for marking web pages for ambient notification much in the style of bookmarking, and the second one extending an email filter to create ambient links for email events. Finally we effects of our experiments in the workplace.
\end{abstract}

Keywords. ambient media, calm technology, world-wide web, linking virtual and physical world, ubiquitous computing

\section{Introduction}

The amount of detailed information available to us is incredible. To reduce this information overload researchers from different areas are developing methods to condense the amount of information. This ranges from simple statistics (e.g. analyzing web access), over filtering mechanisms according to profile (Lieberman 1995) to more sophisticated agent technology using artificial intelligence methods as pointed out by Maes (1994 and 1997). Many of these approaches deliberately trade in the timely context of virtual and real events.

Calm technology and ambient media enable new ways to consume information. Calm technology advocates peripheral awareness of activity in the virtual world or mediated through the virtual world (Weiser and Brown 1995). Ambient media (or ambient displays, Wisneski et al 1998) are means for information presentation in our surroundings. Examples described in the above referenced papers include Jeremijenko's dangling string, and the ambientROOM with water ripples, active wallpaper and ambient sound, showing that ambient media are a rich concept for creation of interfaces between people and virtual worlds, engaging all of the human senses, and blending into our surroundings. Such seamless integration of our environment infor- 
mational space is discussed in the Tangible Bits vision described by Ishii and Ulmer (1997). An interesting aspect of ambient media is to present information in largely abstracted and unmonopolizing ways while preserving the timely context, for example for subconscious awareness of information. Such awareness can for instance give a sense of being connected to others (Holmquist 1998), an important aspect in cooperative workplaces, or a sense of reward as reported by Liechti et al (1998) in their work on social awareness of web site visitors.

Assuming ambient media as highly suited for peripheral information awareness the question is which information to select for ambient presentation, and what kind of ambient medium to use for presentation. This is discussed in Wisneski et al (1998), basically concluding that the decision is not straightforward and that the matching of information and ambient media depends on a wide range of factors. Reported examples of ambient media tend to describe the relation between information and ambient medium in terms of metaphors, for instance for the dangling string presenting network traffic the metaphor of jumping bits is used to describe the meaning of string vibration. The reported examples generally suggest an importance of abstraction in the choice of ambient medium, as less abstract (i.e. more literal) media tend to be perceived as more intrusive.

In this paper we report on investigation of two concepts for matching of information and ambient media. The first one, ambient counterparts, is based on the assumption that much of the information that we handle in our virtual worlds has a natural counterpart in our physical environment, to which it can be related intuitively. In some sense this presents a slight departure from abstraction in the choice of ambient medium. One idea developed in exploration of ambient counterparts is that similar chunks of information will relate to a collection of similar ambient media, supporting an awareness of for instance comparative information. This idea was studied in an application of ambient media for comparative web site awareness employed for group motivation in their workplace.

The second concept for media matching that we report on is to let users themselves create and control ambient links to relate information to ambient media. This may be seen as drastic departure from the concept of calm technology as it requires the user to some extent to attend to technology they should not be consciously aware of. Yet we would argue that provided the creation of ambient links is easy to perform, it will empower users to create personal ambient spaces to handle their information. We describe two tools for user control over ambient links, the first one for marking web pages for ambient notification, and the second one extending an email filter to create ambient links for email events. Prior to the discussion of ambient counterparts in section 3 , and ambient links in section 4 , we briefly describe the underlying ambient media system.

\section{Ambient Media System}

Web-based Architecture. The architecture of the system we built for exploration of ambient counterparts and ambient links is depicted in figure 1 shaded in gray, with a standard web server and a media controller for each ambient medium available. 


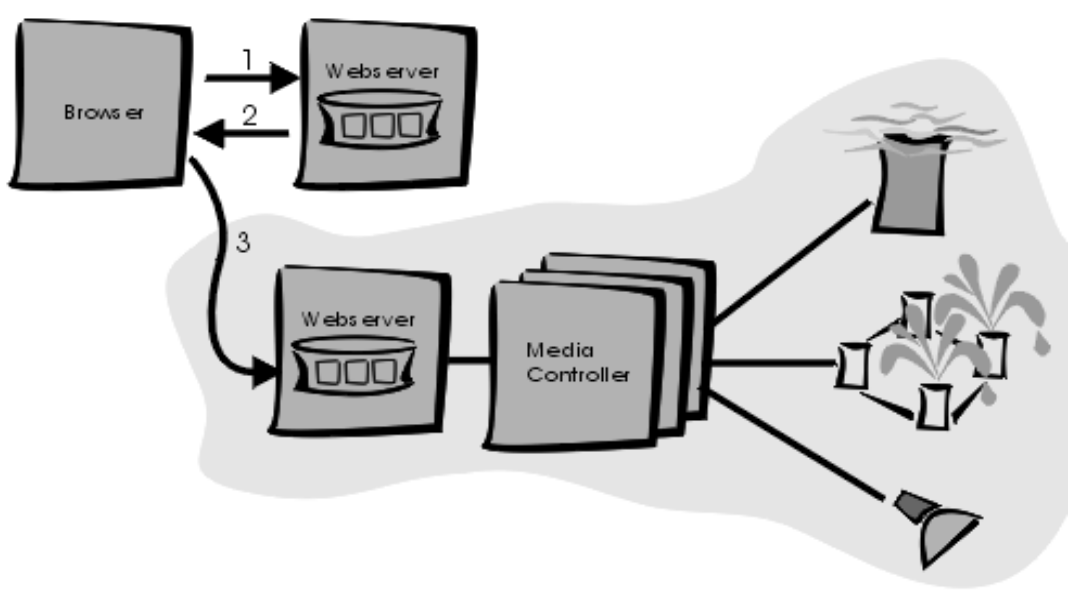

Figure 1: Architecture

A standard web server with CGI is used to receive the requests that control ambient media. The use of web server and CGI allows to write control applications that can be accessed from anywhere. In this architecture, new ambient media can be integrated by simply providing a CGI-script to control it. A media controller can be fully implemented in software as CGI program if the ambient medium is connected by a standard interface (e.g. laser unit connected via the serial port). If this is not the case, control hardware is required in addition to the CGI program (e.g. parallel interface switching power plugs). Depending on the control hardware a CGI program may be parameterized with supported control sequences. The CGI program determines how an ambient medium presents information, and hence the design of such programs is considered part of the design of ambient media rather than application design.

The media controllers are the system abstraction from ambient media, which means that any physical media can be integrated into the system, provided they can be controlled through a defined interface. In the simplest case, very basic devices may be controlled by switching power on and off; more advanced gadgets may be controlled by the power supply (e.g. motor, lights); and rather sophisticated appliances may actually be computer controlled (e.g. serial line controlled laser).

Event Communication. The web server in our architecture is accessed using the standard HTTP-protocol for communication. Virtual events such as web page access, incoming email, calendar events, and modification of web pages, are sent via HTTPGET-request to the web server connected to the media controllers. For clients that access a media controller, based on virtual events, the HTTP-protocol is a reasonable choice as well. In the case of web page access this HTTP-request is automatically sent by the browser while loading the page, because in our approach ambient links are embedded as image in the HTML page (cf. section 4 for description of the ambient links concept). This scenario is illustrated in figure 1: a browser requests a page from a web server containing pages with ambient links (1), the page is sent back to the 
browser (2), containing an IMG-tag with a URL pointing to the media control web server. The browser analyzes the HTML-code and requests all images (3) including the CGI-script that controls the ambient media. The request of the CGI script triggers manipulation of the related ambient medium; it does not return any data to the client. The support for the HTTP-protocol is on most platforms and for major programming languages (Perl on Unix, Visual Basic on Windows, Java) very well what makes the creation of not browser-based clients easy.

\section{Ambient Counterparts of Virtual Information}

Ambient Counterparts. According to our experience much of the information in our virtual spaces has an intuitive and strong relationship to objects in our physical spaces. For instance, product information in a companies web pages can be related to products in a showroom; incoming email from close people can be related to a personal photo gallery; and papers downloaded from a research group's web can be related to posters in the group's office environment, in the case of our research group actually the hallway. We suggest that physical objects in such relationships lend themselves to ambient presentation of related virtual information as its ambient counterpart. For certain relationships between virtual events and real world objects this link may seem obvious, for others it may depend on individual associations, e.g. a postcard of Munich in the office may be associated with home, a great holiday, or $a$ friend living there.

An interesting aspect of the examples stated above is multiplicity: a class of physical objects is related to a class of virtual information. This leads to the consideration that counterparts can be used collectively for an ambient presentation of class-based rather than instance-based information. Consider the email example, then notification of an individual email through, say, brief illumination of the related photo would constitute instance-based information. Use of illumination to show who was in touch over the last week can also be perceived as class-based information (e.g. density of light), providing an overview rather than detail on one instance. Overview is one example, others are comparison of information, and order of events; further information characterizations worth to be investigated is reported in literature on Intelligent Multimedia (e.g. Maybury, 1993).

To study ambient counterparts, we implemented a web site awareness system, in which parts of the web site were related to posters in our hallway. These posters were used to provide an ambient presentation of access to the related pages, to support comparison.

Comparative Web Site Awareness. In this experiment we explored the effect of giving users in a workspace the awareness of other people who are interested in their work. When we have visitors to our office we usually get an impression of what things they are more interested in and which things they do not pay much attention to, as they walk past posters in the hallway, look at different prototypes in the hardware lab, or pick paper copies from our handout material. We also have visitors to our web 
site and it is certainly of interest to get an impression of what these virtual visitors are intersted in. There is a wide range of tools to monitor web site visits, evaluating web server log-files, but only few people use them regularly, because it just enlarges the information overload.

Liechti et al (1998) report on work giving individual users awareness of visitors to their personal web space. In their study, ambient audio is used to notify access to user selected web pages, and they report that such simple notification can be highly motivating to keep web pages interesting and updated. In our study, we extended this concept of web site awareness from the individual to the group.

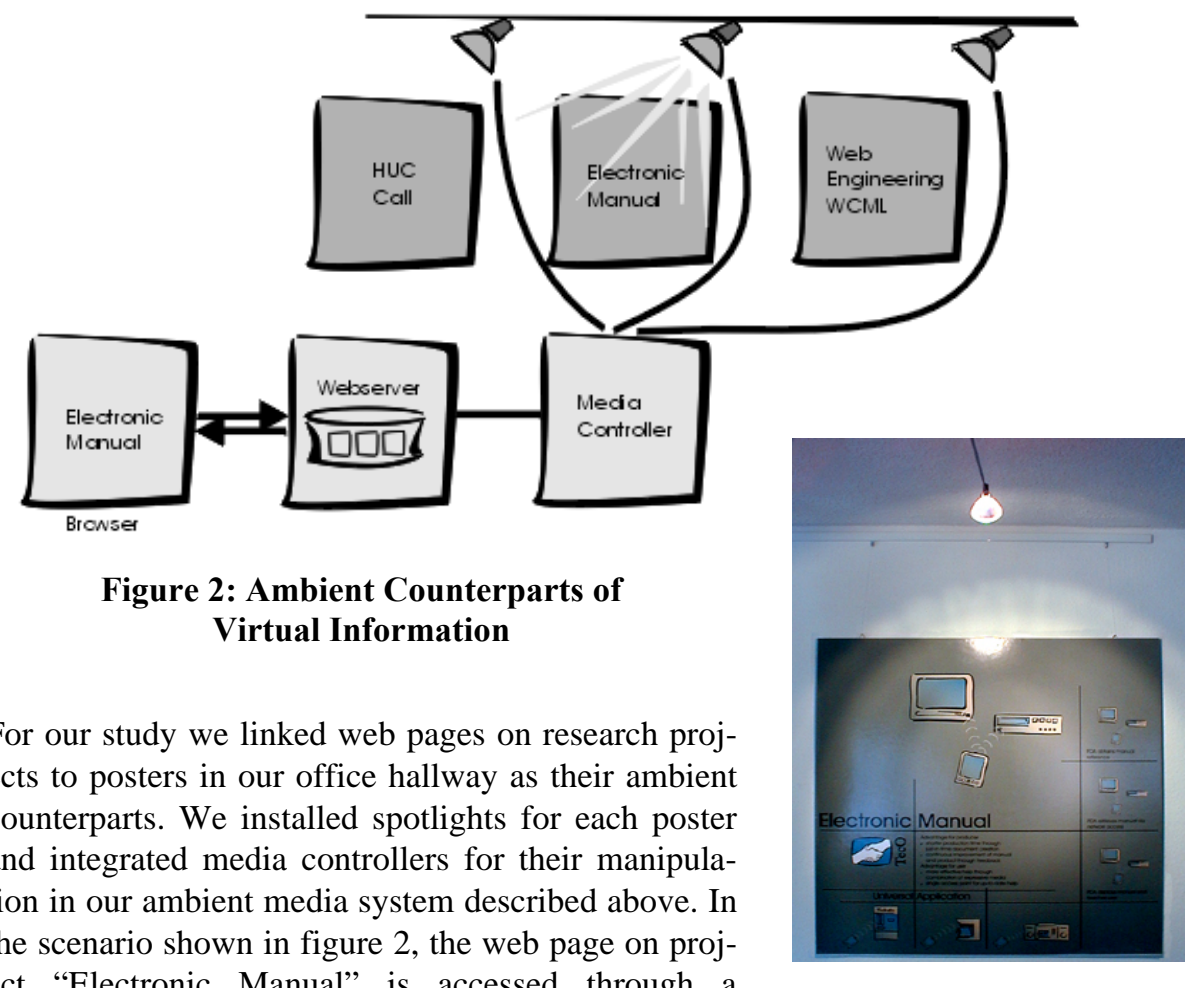
ect "Electronic Manual" is accessed through a browser. This leads to execution of the media controller script, turning the spotlight of the related poster on, to then slowly fade off over time. The photo in figure 2 is a snapshot of the "Electronic Manual" poster, illuminated after access to the project pages in the web. The choice of presentation in this study, slowly fading light, was somewhat arbitrary. Another way to display access activity would for instance be to accumulate hits over time and adjust the light level accordingly. This choice of mapping from data to ambient presentation, and also the choice of how transient or persistent an ambient effect is, remains to be studied.

The setup for ambient web site awareness provided staff and students involved in the respective projects with a good impression of what topics are of interest to our visitors. It also provided feedback on the effect of URL announcements in newsgroups. Most interesting is that the setup quickly gives an impression of how popular 
parts of the web site are in comparison, which may well stimulate competition among project groups to gain popularity for their web pages. Our small study was not suited to derive significant results on such effects but we feel confident to propose comparative web site awareness as a group motivation tool, for example for groups editing electronic journals or other highly dynamic web sites.

\section{User Controlled Ambient Links}

User Controlled Ambient Media. As another aspect of matching virtual information with ambient media we investigated the concept of user controlled ambient media, and developed end user tools for management of ambient links. To require users to decide about usage of ambient media is quite in contrast to the philosophy that users best not be aware at all of the technology that blends into their surroundings. Yet, to have ambient media at one's disposal to create highly individualized ambient spaces seems very intriguing. Also given that the decision on choice of ambient media is far from understood but clearly depends on users' tasks and preferences (cf. Wisneski et al, 1998), it may make sense to defer the decision from design time to run time.

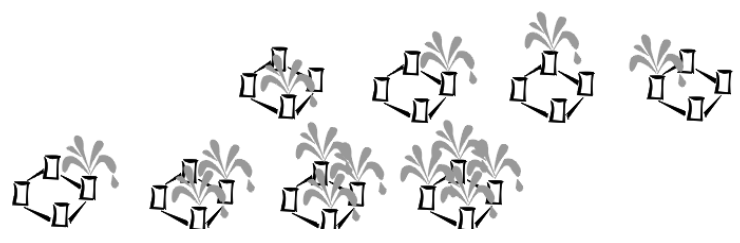

\section{Figure 3: Patterns and Table Fountain}

A critical issue in giving the user control over ambient media is the effort required for creation, modification and deletion of ambient links.

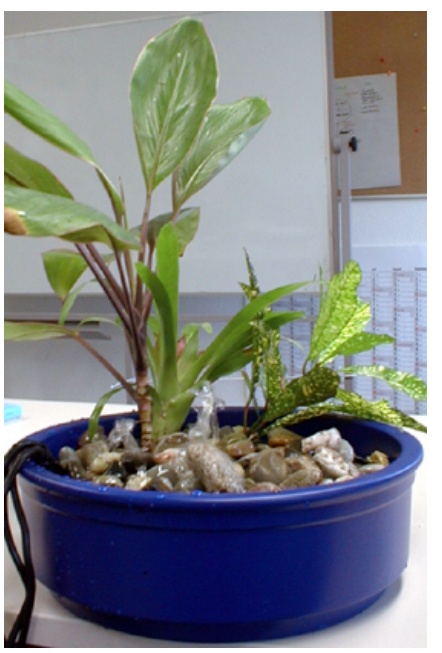
We describe two tools that we developed for managing ambient links, both concerned with provision of easy-to-use mechanisms to link virtual information to ambient media. The first tool is for relating web page access to ambient presentations, and the second one extends an email filter for ambient notification. Both examples uses a small table fountain as ambient medium.

Table Fountain as Ambient Medium. A simple but nevertheless useful and pleasant ambient media is a small fountain placed in our office. It has four pumps that can be switched on and off independently (see figure 3). A PC controls the power plugs over the parallel interface board. The same interface can be used to integrate other devices that are controlled by switching on and off the power, for instance we have also used ultrasonic humidifiers in other demonstrators. The media controller for the fountain is a CGI-program running on the control PC, and generating control information send to 
the interface board over the parallel port. This CGI program has memory of earlier events and of the current state of the fountain, and implements support for different patterns and control effects of the fountain, such as flash, circle, all on, rotate, and add. The effects flash and circle are transient and leave no trace an event has been presented. The effects all on, rotate, and add are less transient, with the effect of an event remaining visible for some time afterward because of the state change.

Marking Web Pages with Ambient Links. The first tool we developed had the goal to mark a web page with an ambient link in a way as easy as marking a web page with a bookmark. The tool allows to browse through a web site to select pages to link with an ambient medium which then is triggered by each access to that page. For instance, in the screenshot shown in figure 4, a workshop web page has been selected to get notification on virtual visits. The tool allows for selection of an ambient media effect, with flash chosen in this case so that access to the specified page will let the fountain bubble briefly. Obviously, the tool is geared to control of the table fountain described above but it is straightforward to envision a generalized management tool supporting selection among multiple ambient media, and media-specific selection of effects.

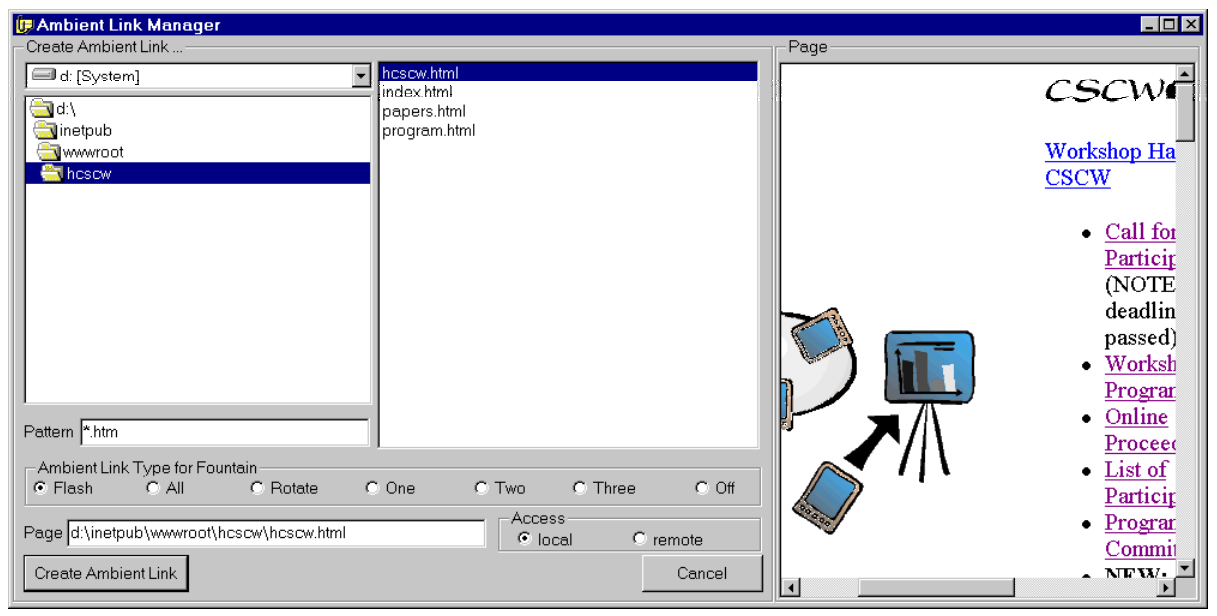

Figure 4: Ambient Link Manager

After selection of web page as information source and the ambient presentation effect, ambient links are established by pushing the create button. This actually leads to insertion of a line of HTML-code to the file. This line contains a call to the CGI-script on the web server controlling the ambient media:

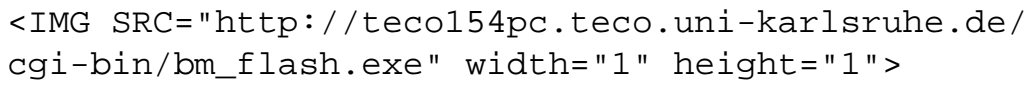

Note that insertion of this line in an arbitrary web page in the world-wide web would result in our table fountain bubbling for a moment whenever that page is accessed! 
The effect is not restricted to local web pages but of course the creation of ambient links is restricted to pages for which the user has modification rights.

It also has to be noted that of course different pages can be linked to the same ambient medium, and one and the same page can have links to different ambient media. The ambient link management tool also provides the functionality to remove ambient links from web pages, that is to remove the inserted image tag.

Ambient Email Notification. The tool for ambient email notification is based on the elm mail filter system. The tool allows the user to create rules that call a media controller (i.e. a CGI-script). In the condition part of the rule subject and sender can be selected to identify mails, and in the action part the user can specify the ambient effect to be triggered by an incoming email that meets the condition. The technical realization is done using a Perl-script that calls the CGI-program controlling the ambient medium. An example of a rule generated by this tool is:

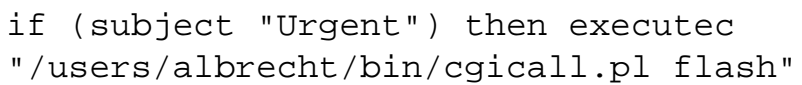

The effect of this rule is that email containing Urgent in the subject line causes the table fountain to bubble for a moment (flash).

Further User Controls. At the moment we a working on two further tools, one to map calendar events to ambient media and another one to monitor other web pages and give notification by physical output. The calendar editor is straightforward, additional to the time when the user should be notified it will be possible to select the system that is used to notify the user. The channel editor has enhanced functionality and can be used to monitor any web page. The user selects the URL to monitor the event they are interested in (e.g. change of page or certain keyword appearing on page), and the ambient effect that should be produced if the condition is true. Execution will be based on Unix system cron jobs.

\section{Conclusion and Future Work}

We have explored two ideas for matching of virtual information and ambient media, with a range of interesting findings. First, investigating ambient counterparts of virtual information we came to consider class-information rather than instanceinformation for ambient display, enabling for example overview and comparison. We applied the idea of ambient comparative information in a study that extended web site awareness from the individual to the group, a concept we believe has potential for group motivation in the workplace, and for better integrating web sites or other informational spaces as virtual extension of work environments. As second idea, we considered user control over links between virtual information and ambient media, and described tools that make ambient linking as simple as bookmarking. We would argue that with such tools users can be empowered to create personal ambient spaces to handle information in entirely new ways. 
While designing the prototypes we realized that for various virtual events the link to real world objects is easily conceivable by users. But still for numerous virtual events links to real world objects are perceived differently by different people, for one it seams straightforward while for another one it is very artificial. Therefore we think letting the user decide were to place the ambient links is a good decision. The link management must be very easy to handle by the user to make it possible to change ambient media when changing the foreground task to avoid interference between foreground task and ambient display as discussed by Wisneski et al. (1998).

After getting used to the ambient media setup this becomes quickly valuable to us; the peripheral information space becomes part of the work environment. So far we have not carried out a formal user study but we will describe some observation we made while using the prototypical system. We found out that depending on the things we were working on, the focus and goals we had, and also the personal form we used the system differently. Firstly the mapping chosen depended very much on what we were interested in, e.g. after announcing a conference call to news groups we chose a transient mapping of this page to the fountain or when waiting for email from somebody while reading or doing some other work not at the computer this was mapped persistent to an output. Secondly the perception of the events depended mainly on the personal condition, when working under time pressure the ambient information was hardly realized whereas when bored an ambient indication led people to look at the log-file of the server to find out who it was.

In the setup of the first experiment we found out that the inclusion of ambient media control affects the design and structure of the information that is display on the web pages. For this approach it is certainly a reasonable decision to design ambient media together with the web pages.

The effect that virtual events become more real at the workplace has interesting implications. First the motivation can be increased by feeling every now and then that someone is interested in your work and in the thinks you a displaying on your website, this is also reported by Liechti et al. (1998). Virtual events become more a part of your work life, colleagues sharing the office asked once your fountain didn't bubble the whole morning - is your server down? A setup as described in the first experiment introduces implicit competition, by making access to certain web pages public. This can have positive effects and lead to discussion but in highly competitive environment this could be negative, too.

In the future we plan to create a dynamic mapping of media according to the preferences and context of the user. We are also looking for new application domains and working on the support of more general control system using power line control (e.g. $\mathrm{X} 10$ ). Furthermore we think there is need for a model that describes mappings of information to ambient media with respect to persistence and transience based on the communication goals. 


\section{References}

1. Holmquist, L. E., Wigström, J., Falk, J. (1998). The Hummingbird: Mobile Support for Group Awareness. Demo at the Conference on CSCW'98. Seattle, 14-16. November 1998. To appear in Handheld CSCW, Personal Technologies (special issue), vol. 3 no. 1, 1999.

2. Ishii, H. and Ullmer, B (1997). Tangible Bits: Towards Seamless Interfaces between People, Bits and Atoms, in Proceedings of CHI'97 (Atlanta GA, March 1997), ACM Press, 234241.

3. Lieberman, H. (1995). Letizia: An Agent That Assists Web Browsing, Proceedings of the 1995 International Joint Conference on Artificial Intelligence, Montreal, Canada, August 1995.

4. Liechti, O., Siefer, N., Ichikawa, T. (1998). A Non-obtrusive User Interface for Increasing Social Awareness on the World Wide Web. Workshop on Handheld CSCW on the CSCW'98, http://www.teco.edu/hcscw/papers.html. Seattle, USA (November 14, 1998). To appear in Handheld CSCW, Personal Technologies (special issue), vol. 3 no. 1, 1999.

5. Maes, P. Agents that Reduce Work and Information Overload, Communications of the ACM, Vol. 37, No. 7, (July 1994).

6. Maes, P. (1997). Interview: Pattie Maes on Software Agents: Humanizing the Global Computer. IEEE Internet Computing. July-August 1997.

7. Maybury, M. (1993). Intelligent Multimedia Interfaces. AAAI Press. Menlo Park, USA.

8. Weiser, M., Brown, J.S. (1996). Designing Clam Technology. PowerGrid Journal, v 1.01, http://powergrid.electriciti.com/1.01 (July 1996).

9. Wisneski, G., Ishii, H., Dahley, A., Gorbet, M., Brave, S., Ullmer, B., Yarin, P. (1998). Ambient Display: Turning Architectural Spache into an Interface between People and Digital Information. In: Proceedings of the First International Workshop on Cooperative Buildings (CoBuild'98), Darmstadt, Germany (February 25-26, 1998). Lecture Notes in Computer Science, Vol. 1370. Springer - Verlag, Heidelberg. 